\title{
The line method combined with spectral chebyshev for space-time fractional diffusion equation
}

\author{
I. K. Youssef ${ }^{1, ~ *}$, A. M. Shukur ${ }^{2}$ \\ ${ }^{1}$ Department of Mathematics, Ain Shams University, Cairo, Egypt \\ ${ }^{2}$ Department of Applied Mathematics, University of Technology, Baghdad, Iraq
}

Email address:

kaoud22@hotmail.com (I. K. Youssef), ahmhshald@yahoo.com (A. M. Shukur)

\section{To cite this article:}

I. K. Youssef, A. M. Shukur. The Line Method Combined with Spectral Chebyshev for Space-Time Fractional Diffusion Equation. Applied and Computational Mathematics. Vol. 3, No. 6, 2014, pp. 330-336. doi: 10.11648/j.acm.20140306.17

\begin{abstract}
The Method of Lines Combined with Chebyshev Spectral Method respect to weighted residual (Collocation Points) for Space-Time fractional diffusion equation is considered, the direct way will be used for approximating Time fractional and the expiation of shifted first kind of Chebyshev polynomial will be used to approximate unknown functions, the structure of the systems and the matrices will be fund, the algorithm steps is illustrated, The tables and figures of the results of the implementation by using this method at different values of fractional order will be shown, with the helping of programs of matlab.
\end{abstract}

Keywords: Space-Time Fractional Diffusion Equation, Chebyshev-Spectral Method, Finite Difference Method

\section{Introduction}

Diffusion equation provide an important tool for modeling a numerous problems in engineering, physics and others science, the generalization of the integer order diffusion equation to space fractional order, time fractional order or more general is the space-time fractional order diffusion equation (S-TFPDE), is more important to study it and to find the easier ways to solve it.

When time dependent PDEs are solved numerically by spectral methods $[1,2]$, the pattern is usually the same: employ spectral treatment to space dependency and use finite difference in time or leave the time dependency to obtain a system of ordinary differential equations (ODE) in time. In this paper, S-TFPDE has been considered

$$
\begin{gathered}
\frac{\partial^{\alpha} u(x, t)}{\partial t^{\alpha}}=\frac{\partial^{\beta} u(x, t)}{\partial x^{\beta}}+g(x, t) ; \\
0<\alpha \leq 1<\beta \leq 2,0 \leq x \leq L, 0 \leq t \leq T,
\end{gathered}
$$

With initial and boundary conditions

$$
\begin{gathered}
u(0, t)=u(L, t)=0 ; \text { for } 0 \leq t \leq T, \\
u(x, 0)=f(x) ; \text { for } 0 \leq x \leq L .
\end{gathered}
$$

The idea of the treatment in this paper depends on the method of discretization in time (the method of lines) [3]. In this method the time interval $[0, T]$ is divided into $M$ subintervals $I_{1}, I_{2}, \cdots, I_{M} \quad\left(I_{j}=\left[t_{j-1}, t_{j}\right], j=1,2, \cdots, M\right)$ of length $\tau=\frac{T}{M}$, and the problem is transformed to solve fractional boundary value problem along each time level. For the fractional boundary value problem a spectral collocation method is employed.

Spectral method [1,2] involve seeking the solution to differential equations in terms of a series of known, smooth functions. Spectral methods may be viewed as an extreme development of the class of discretization schemes for differential equations known generally as the method of weighted residuals (WRM). The key elements of the WRM are the trial functions and the test functions. The trial functions are used as the basis functions for a truncated series expansion of the solution. The test functions are used to ensure that the differential equation is satisfied as closely as possible by the truncated series expansion. The choice of test function distinguishes which used spectral schemes, is collocation. In the collocation approach the test functions are the translated Dirac delta functions centered at the collocation points. The treatment considered in this work depends on the use of the collocation approach.

Collocation

The core of the collocation method is definition of the residue function and the collocation points. Consider the boundary value problem, $[4,5]$. 


$$
\begin{gathered}
\mathcal{L} \varphi=f, a \leq x \leq b, \\
\varphi(a)=\varphi(b)=0 .
\end{gathered}
$$

Where $\mathcal{L}$ is any operator. An approximate solution $\psi$ will not, in general, satisfy (3) exactly, and associated with such an approximate solution is the residual defined by

$$
R(\psi)=\mathcal{L} \psi-f
$$

It is clear that if $\psi$ is the exact solution then $R(\psi)=0$.

In the collocation the trial functions, $\left\{\varphi_{j}\right\}_{j=1}^{N}$, are used as the basis functions for a truncated series expansion of the solution. $\psi^{N}(x)=\sum_{j=0}^{N} C_{r} \varphi_{j}(x)$.

The shifted Chebeshev polynomials $T_{N}^{*}(x)$ are used as the basis function $\varphi_{j}$, and the Gauss-Labatto points

$x_{j}=0.5 \cos \left(\frac{j \pi}{N}\right)+0.5, j=0,1, \cdots, N$ are used as the collocation points. Accordingly,

$$
R\left(\psi^{N}\left(x_{j}\right)\right)=\mathcal{L} \psi^{N}\left(x_{j}\right)-f, j=0,1, \cdots, N .
$$

Represents a system of $(N+1)$ algebraic equations in $(N+1)$ unknowns $C_{r}$. Determination of $C_{r}$ enables us to write the approximate solution as a linear combination of the bases functions.

\section{The Caputo's Fractional Derivatives $\left({ }_{0}^{C} D_{x}^{\alpha}\right),[2]$}

For any real function $\mathrm{f}(\mathrm{x}) \in C^{m}$, the Caputo fractional derivative of order $\alpha$ is:

$$
{ }_{0}^{c} D_{x}^{\alpha} f(x)=\frac{1}{\Gamma(m-\alpha)} \int_{0}^{x}(x-s)^{m-\alpha-1}\left(\frac{d}{d s}\right)^{m} f(s) d s,
$$

where $x>0 ; m-1<\alpha \leq m$

$$
D_{x}^{\alpha} x^{p}= \begin{cases}0 & \text { if } p<\alpha \\ 0 & \text { if } x^{p} \text { constant } \\ \frac{\Gamma(p+1)}{\Gamma(p-\alpha+1)} x^{(p-\alpha)} & \text { if } p \geq \alpha\end{cases}
$$

The Caputo fractional derivative operator is linear in the sense that for any two constants A, B and any two functions $\{\mathrm{f}(\mathrm{x})$ and $\mathrm{g}(\mathrm{x})\} \in C^{m}$, one can write

$$
{ }_{0}^{C} D_{x}^{\alpha}\{A f(x) \mp B g(x)\}=A_{0}^{C} D_{x}^{\alpha} f(x) \mp B_{0}^{C} D_{x}^{\alpha} g(x)
$$

\section{Chebyshev Polynomials CPs $[1,8,9]$}

Chebyshev polynomials (CPs) of the first kind denoted by $T_{n}(x),-1 \leq x \leq 1, n=0,1,2, \cdots$ and defined as

$$
\begin{gathered}
T_{0}(x)=1, T_{1}(x)=x \text { and } \\
T_{n}(x)=2 x T_{n-1}(x)-T_{n-2}(x), n \geq 2 .
\end{gathered}
$$

The significance can be immediately appreciated from the fact that the function $\cos (n \theta)$ is a Chebyshev polynomial function of $\cos (\theta)$. Specifically, for $n \geq 0$

$$
\cos (n \theta)=T_{n}(\cos (\theta))
$$

The analytic form of the Chebyshev polynomial $T_{n}(x)$ of degree $n$ is given by

$$
\begin{gathered}
T_{n}(x)=\frac{n}{2} \sum_{k=0}^{\left\lfloor\frac{n}{2}\right\rfloor}(-1)^{k} \frac{\Gamma(n-k)}{k ! \Gamma(n-2 k+1)}(2 x)^{(n-2 k)} \\
T_{0}(x)=1 .
\end{gathered}
$$

where $\left\lfloor\frac{n}{2}\right\rfloor$ is the floor of $\frac{n}{2}$, (the integer part of $\frac{n}{2}$ ).

It is clear that CP $T_{n}(x)$ is a polynomial of degree $n$, The CP's have $n$ zeros in the interval $[-1,1]$, given by

$$
x_{j}=\cos \left(\frac{2(j+1)}{n} \frac{\pi}{2}\right), \mathrm{j}=0,1,2, \ldots, \mathrm{n}-1,
$$

The CP's have $n+1$ extremes points in same interval $[-1,1]$, given by

$$
x_{j}=\cos \left(\frac{j \pi}{n}\right), \quad \mathrm{j}=0,1,2, \ldots, \mathrm{n} .
$$

The CP $T_{n}(x)$ are orthogonal functions in the sense that

$$
\int_{-1}^{1} \frac{T_{m}(x) T_{n}(x)}{\sqrt{1-x^{2}}} d x=\left\{\begin{array}{cc}
0 & m \neq n \\
\frac{\pi}{2} & m=n \neq 0 \\
\pi & m=n=0
\end{array} .\right.
$$

In order to use CPs on intervals of the form $[a, b]$ other than the interval $[-1,1]$ one can use the change of the variable $x=2\left(\frac{t-a}{b-a}\right)-1$, which transforms the points $t \in[a, b]$ to $x \in[-1,1]$, conversely, $t=\left(\frac{b-a}{2}\right) x+\left(\frac{b+a}{2}\right)$, transforms the points $x \in[-1,1]$ to $t \in[a, b]$

Accordingly, the shifted Chebyshev polynomials (SHCP), $T_{n}^{*}(x)$ of degree $\mathrm{n}$ on interval $[\mathrm{a}, \mathrm{b}]$ can be written as

$$
\begin{gathered}
T_{n}^{*}(x)=T_{n}\left[2\left(\frac{x-a}{b-a}\right)-1\right], \text { for } n \in \mathbb{N}, n \geq 1, \\
T_{0}^{*}(x)=1 .
\end{gathered}
$$

Where $T_{n}(x)$ is the first kind CP defined in (11).

\section{Fractional Derivatives of SHCP}

The Caputo's Fractional derivative of SHCP defined in (14), of order $>0$, can be written with the helping of properties in (6-8) and the definition of the SHCP formula (12).

Lemma: The Caputo's fractional derivatives of order $m-1<\beta<m, m=\lceil\beta\rceil$, of shifted Chebyshev polynomial of degree $n, 0 \neq n \in \mathbb{N}, n \geq 1$, on interval [a,b] is

$$
\begin{gathered}
{ }_{a}^{C} D_{x}^{\beta} T_{n}^{*}(x)=\frac{n}{2}\left(\frac{2}{b-a}\right)^{m}(x-a)^{m-\beta} * \\
\sum_{k=0}^{\left\lfloor\frac{n}{2}\right\rfloor}(-1)^{k} \frac{\Gamma(n-k)}{\Gamma(k+1)}(2)^{(n-2 k)} M(x ; n, k, m),
\end{gathered}
$$




$$
\begin{gathered}
M(x ; n, k, m) \\
=\left\{\begin{array}{cc}
0, & m>n-2 k \\
\frac{1}{\Gamma(m-\beta+1)^{\prime}}, & m=n-2 k \\
\sum_{r=0}^{n-2 k-m} \frac{(-1)^{r+n-2 k-m}}{\Gamma(r+m-\beta+1) \Gamma(n-2 k-m-r+1)} & m<n-2 k \\
*\left[2\left(\frac{x-a}{b-a}\right)\right]^{r}, &
\end{array}\right.
\end{gathered}
$$

Proof

Consider the Caputo's fractional derivatives of power functions and the linearity property

$$
D_{x}^{\beta} A x^{p}=\left\{\begin{array}{cc}
0, & \text { if } p=0 \\
\frac{A \Gamma(p+1)}{\Gamma(p-\beta+1)} x^{(p-\beta)}, & \text { if } p \geq \beta .
\end{array} \text { where } A\right. \text { is constant }
$$

Write formula (11) of SHCP and applying the Caputo's derivative on it with using the linearity property, yields

$$
\begin{gathered}
{ }_{a}^{C} D_{x}^{\beta} T_{n}^{*}(x)={ }_{a}^{c} D_{x}^{\beta} T_{n}\left[2\left(\frac{x-a}{b-a}\right)-1\right] \\
=\frac{n}{2} \sum_{k=0}^{\left\lfloor\frac{n}{2}\right\rfloor}(-1)^{k} \frac{\Gamma(n-k)(2)^{(n-2 k)}}{k ! \Gamma(n-2 k+1)}{ }_{a}^{c} D_{x}^{\beta} \\
{\left[2\left(\frac{x-a}{b-a}\right)-1\right]^{n-2 k}}
\end{gathered}
$$

The Caputo's derivative part in this formula can be determined as follows, Use the definition of CFD,

$$
{ }_{a}^{C} D_{x}^{\beta}\left[2\left(\frac{x-a}{b-a}\right)-1\right]^{n-2 k}={ }_{a} J_{x}^{m-\beta} D_{x}^{m}\left[2\left(\frac{x-a}{b-a}\right)-1\right]^{n-2 k},
$$

then the following three cases are obtained

$$
\begin{gathered}
\text { i-If } m>n-2 k, D_{x}^{m}\left[2\left(\frac{x-a}{b-a}\right)-1\right]^{n-2 k}=0, \\
\text { ii-If } m=n-2 k, D_{x}^{m}\left[2\left(\frac{x-a}{b-a}\right)-1\right]^{n-2 k} \\
=(n-2 k) !\left(\frac{2}{b-a}\right)^{n-2 k},
\end{gathered}
$$

Which is the constant term and $m-\alpha \geq 0$, using R-L FI to opten,

${ }_{a} J_{x}^{m-\beta} D_{x}^{m}\left[2\left(\frac{x-a}{b-a}\right)-1\right]^{n-2 k}=(n-2 k) !\left(\frac{2}{b-a}\right)^{n-2 k} *$

$\frac{\left(^{(x-a)^{(m-\beta)}}\right.}{\Gamma(m-\beta+1)}$,

Thus

$$
\begin{aligned}
{ }_{a}^{C} D_{x}^{\beta}\left[2\left(\frac{x-a}{b-a}\right)\right. & -1]^{n-2 k} \Gamma(n-2 k+1) * \\
& *\left(\frac{2}{b-a}\right)^{m} \frac{(x-a)^{(m-\beta)}}{\Gamma(m-\beta+1)}
\end{aligned}
$$

i-If $m \leq n-2 k, \quad D_{x}^{m}\left[2\left(\frac{x-a}{b-a}\right)-1\right]^{n-2 k}$

$$
=\frac{\Gamma(n-2 k+1)}{\Gamma(n-2 k-m+1)}\left(\frac{2}{b-a}\right)^{m}\left[2\left(\frac{x-a}{b-a}\right)-1\right]^{n-2 k-m} .
$$

Since $n-2 k-m \in \mathbb{Z}^{+}$and $n-2 k \geq 0$, by applying the binomial expantion to the last term and consequently using the R-L FI term by term, there is

$$
\begin{aligned}
& a J_{x}^{m-\beta}\left[2\left(\frac{x-a}{b-a}\right)-1\right]^{n-2 k-m} \\
& =\left(\frac{2}{b-a}\right)^{n-2 k-m} J_{x}^{m-\beta}\left[(x-a)-\left(\frac{b-a}{2}\right)\right]^{n-2 k-m}, \\
& =\left(\frac{2}{b-a}\right)^{n-2 k-m} a J_{x}^{m-\beta}\left[\sum_{r=0}^{n-2 k-m}\left(\begin{array}{c}
n-2 k-m \\
r
\end{array}\right)(x-)^{r} *\right. \\
& \left.*\left(-\frac{b-a}{2}\right)^{n-2 k-m-r}\right]
\end{aligned}
$$

$=(-1)^{n-2 k-m} \sum_{r=0}^{n-2 k-m} \frac{\Gamma(n-2 k-m+1)}{\Gamma(r+1) \Gamma(n-2 k-m-r+1)}$

$*(-1)^{r}\left(\frac{2}{b-a}\right)^{r} J_{x}^{m-\beta}(r-a)^{r}$,

$=\Gamma(n-2 k-m+1)(x-a)^{m-\alpha} *$

$\sum_{r=0}^{n-2 k-m} \frac{(-1)^{r+n-2 k-m}}{\Gamma(n-2 k-m-r+1) \Gamma(r+m-\beta+1)}\left(\frac{2}{b-a}\right)^{r} *\left[2\left(\frac{x-a}{b-a}\right)-1\right]^{r}$,

Thus obtained

$$
\begin{gathered}
{ }_{a}^{C} D_{x}^{\beta}\left[2\left(\frac{x-a}{b-a}\right)-1\right]^{n-2 k}= \\
=\Gamma(n-2 k+1)\left(\frac{2}{b-a}\right)^{m}(x-a)^{m-\beta} *
\end{gathered}
$$

$$
\text { * }\left\{\begin{array}{cc}
\frac{0,}{1} \frac{1}{\Gamma(m-\beta+1)^{\prime}} & m>n-2 k, \\
\sum_{r=0}^{n-2 k-m} \frac{(-1)^{r+n-2 k-m}}{\Gamma(r+m-\beta+1) \Gamma(n-2 k-m-r+1)} & m<n-2 k . \\
*\left[2\left(\frac{x-a}{b-a}\right)\right]^{r}, &
\end{array}\right.
$$

Which completes the prove

\section{Description of the Method}

The considered method has two steps, the first step is using the direct way to approximate the time fractional part, the diffusion equation will be system of space fractional ordinary differential equations formula (17), the second step is using the shifted Chebyshev polynomials to approximate the unknown functions then, furmula(17) yield the system of algebraic equation formula (22) with unknown coefficients. Using the spectral method with the weighted residual (collocation method) to find the unknown coefficients.

Consider the space-time fractional diffusion equation

$$
\frac{\partial^{\alpha} u(x, t)}{\partial t^{\alpha}}=\frac{\partial^{\beta} u(x, t)}{\partial x^{\beta}}+g(x, t) \quad ; \quad 0<\alpha \leq 1<\beta \leq 2 \text {, }
$$
$0 \leq x \leq L, 0 \leq t \leq T$, respect to initial and boundary condition 
I.C $u(x, 0)=f(x) ; \quad 0 \leq x \leq L$,

B.C $u(0, t)=u(L, t)=0 ; 0 \leq t \leq T$,

To solve S-TFPDE, and it is clearly to show that $u^{k}=u\left(x, t_{k}\right), k=0,1, \cdots, M-1,1<M \in \mathbb{N}, \quad$ is the numerical function at time-step $t_{k}=k \Delta t, \Delta t=\frac{T}{M}$.

The direct way (finite difference method for Caputo's fractional derivatives) is

$$
\begin{gathered}
{ }^{C} D_{t}^{\alpha} u\left(x, t_{k+1}\right) \cong \omega \sum_{j=0}^{k} b_{j}\left(u^{k-j+1}-u^{k-j}\right)+O(\tau), \\
0<\alpha \leq 1, \\
{ }^{C} D_{t}^{\alpha} u^{k+1}=\omega\left[\sum_{j=0}^{k-1}\left(b_{j+1}-b_{j}\right) u^{k-j}+u^{k+1}-b_{k} u^{0}\right] \\
b_{j}=(j+1)^{1-\alpha}-j^{1-\alpha}, \omega=\frac{\tau^{-\alpha}}{\Gamma(2-\alpha)},
\end{gathered}
$$

for all $x \in[0, L], g^{k}=u\left(x, t_{k}\right)$, so that one can rewrite the $\mathrm{S}$-TFPDE eq(1) by using the approximation of time fractional as

$$
\begin{aligned}
u^{k+1}(x)=\left(\omega D_{x}^{\beta}\right. & \left.+1-b_{1}^{\alpha}\right) u^{k}(x) \\
& +\sum_{j=1}^{k-1}\left(b_{j}^{\alpha}-b_{j+1}^{\alpha}\right) u^{k-j}(x) \\
& +b_{k}^{\alpha} u^{0}(x) \\
& +\omega g^{k}(x)
\end{aligned}
$$

In general the idea of the Spectral Method(SM), [6, 7], is finding the trial approximation for the unknown functions $u^{k}(x)$, by using known basis and assuming this unknown functions equal finite sum of this basis, (in this paper, shifted Chebyshev polynomials have been used), as

$$
u_{N}^{k}(x)=\sum_{r=0}^{n} C_{r}^{k} T_{r}^{*}(x), \text { where } n \in \mathbb{N}, n \geq 1,
$$

Where $k=0,1, \cdots, M-1,1<M \in \mathbb{N}$, it is well known formula (17) can be written as $M\left[u_{N}^{k}(x)\right]=$ $G\left[g^{k}(x)\right]$, where $\left(\left(x, t_{k}\right) \in D\right.$, and $\mathrm{M}$ denoted an operator which maps set of the functions $\mathrm{U}$ into set of $\mathrm{G}$, s.t $u^{k} \in \mathrm{U}$, $g^{k} \in \mathrm{G}$ and $\mathrm{D}$ is the prescribed domain, since the approximate solutions given in eq(18) will be not (in general) satisfy (1) exactly, then we define the residual $R_{n}\left(x, C^{k}\right)$ as

$$
R_{n}\left(x, C^{k}\right)=M\left[u_{N}^{k}(x)\right]-G\left[g^{k}(x)\right],
$$

The residual depended on the coefficients $C^{k}$.

Now the problem is, how one can find $(n+1)$ coefficients $C_{r}^{k}$ in equation (19), to find the solutions in equation(18)?

The answer of this question is, by using the weighted residual method.

Weighted Residuals Methods (WRM)[4,5], in this method one has many ways to define weighted function on D to make the weighted integral on the D equal zero, in this work the collocation method is used,

Defined $W(x)=\left\{\begin{array}{cc}1 & \text { at the collocation points } \\ 0 & \text { otherwise }\end{array}\right\}$, now one can write

$$
\int_{D} W\left(x_{i}\right) R_{n}\left(x_{i}, C^{k}\right)=0,
$$

where $x_{i}, i=0,1, \cdots, N-1$, are the collocation points of Chebyshive which are given in eq(12), from the definition of weighted function $W(x)$ one gets

$$
\begin{gathered}
R_{n}\left(x_{i}, C^{k}\right)=M\left[u_{N}^{k}\left(x_{i}\right)\right]-G\left[g^{k}\left(x_{i}\right)\right], \text { or } \\
M\left[u_{N}^{k}\left(x_{i}\right)\right]=G\left[g^{k}\left(x_{i}\right)\right] .
\end{gathered}
$$

Now from equations (17), (18), and eq(21) one can rewrites the formulas (17) as

$$
\begin{aligned}
\sum_{r=0}^{n} C_{r}^{k+1} T_{r}^{*}(x) & =\sum_{r=0}^{n} C_{r}^{k}\left(\omega D_{x}^{\beta}+1-b_{1}^{\alpha}\right) T_{r}^{*}(x) \\
& +\sum_{j=1}^{k-1}\left[\sum_{r=0}^{n} C_{r}^{k-j}\left(b_{j}^{\alpha}-b_{j+1}^{\alpha}\right) T_{r}^{*}(x)\right] \\
& +\sum_{r=0}^{n} C_{r}^{0} b_{k}^{\alpha} T_{r}^{*}(x)+g^{k+1}(x)
\end{aligned}
$$

From definition of residual eq(21) and the colocation points eq(12), one can obtain a linear system of algebraic equations in the unknowns $\left\{C_{r}^{k+1}\right\}_{r=0}^{n}$ in the form

$$
A^{*} C_{r}^{k+1}=b \text {, where } A C_{r}^{0}=F .
$$

Where the right hand side $b$ is defined explicitly in terms of known data in the previous time levels.

$$
b=A C_{r}^{k}+\sum_{j=1}^{k-1} H^{j} C_{r}^{k-j}+Y C_{r}^{0}+G^{k+1}(x),
$$

$$
C_{r}^{k+1}=\left[\begin{array}{c}
C_{0} \\
C_{1} \\
\vdots \\
C_{n}
\end{array}\right]^{k+1} \quad, k=0,1, \cdots, \in \mathbb{N}
$$

$G^{k+1}=\left[\begin{array}{c}\omega g\left(x_{0}\right) \\ \omega g\left(x_{1}\right) \\ \vdots \\ \omega g\left(x_{n}\right)\end{array}\right]^{k+1}, k=0,1, \cdots, \in \mathbb{N}$.

$H^{j}=\left[\begin{array}{cccc}\left(b_{j}^{\alpha}-b_{j+1}^{\alpha}\right) T_{0}^{*}\left(x_{0}\right) & \left(b_{j}^{\alpha}-b_{j+1}^{\alpha}\right) T_{1}^{*}\left(x_{0}\right) & \cdots & \left(b_{j}^{\alpha}-b_{j+1}^{\alpha}\right) T_{n}^{*}\left(x_{0}\right) \\ \left(b_{j}^{\alpha}-b_{j+1}^{\alpha}\right) T_{0}^{*}\left(x_{1}\right) & \left(b_{j}^{\alpha}-b_{j+1}^{\alpha}\right) T_{1}^{*}\left(x_{1}\right) & \cdots & \left(b_{j}^{\alpha}-b_{j+1}^{\alpha}\right) T_{n}^{*}\left(x_{1}\right) \\ \vdots & \vdots & \vdots & \vdots \\ \left(b_{j}^{\alpha}-b_{j+1}^{\alpha}\right) T_{0}^{*}\left(x_{n}\right) & \left(b_{j}^{\alpha}-b_{j+1}^{\alpha}\right) T_{1}^{*}\left(x_{n}\right) & \cdots & \left(b_{j}^{\alpha}-b_{j+1}^{\alpha}\right) T_{n}^{*}\left(x_{n}\right)\end{array}\right]$

$A^{*}=\left[\begin{array}{cccc}T_{0}^{*}\left(x_{0}\right) & T_{1}^{*}\left(x_{0}\right) & \cdots & T_{n}^{*}\left(x_{0}\right) \\ T_{0}^{*}\left(x_{1}\right) & T_{1}^{*}\left(x_{1}\right) & \cdots & T_{n}^{*}\left(x_{1}\right) \\ \vdots & \vdots & \vdots & \vdots \\ T_{0}^{*}\left(x_{n}\right) & T_{1}^{*}\left(x_{n}\right) & \cdots & T_{n}^{*}\left(x_{n}\right)\end{array}\right]$,

$C_{r}^{0}=\left[\begin{array}{c}C_{0} \\ C_{1} \\ \vdots \\ C_{n}\end{array}\right]^{0}, \quad F=\left[\begin{array}{c}f\left(x_{0}\right) \\ f\left(x_{1}\right) \\ \vdots \\ f\left(x_{n}\right)\end{array}\right]$ 
$A=\left[\begin{array}{ccc}\left(\omega D_{x}^{\beta} T_{0}^{*}+T_{0}^{*}-b_{1}^{\alpha} T_{0}^{*}\right)\left(x_{0}\right) & \cdots & \left(\omega D_{x}^{\beta} T_{n}^{*}+T_{n}^{*}-b_{1}^{\alpha} T_{n}^{*}\right)\left(x_{0}\right) \\ \left(\omega D_{x}^{\beta} T_{0}^{*}+T_{0}^{*}-b_{1}^{\alpha} T_{0}^{*}\right)\left(x_{1}\right) & \cdots & \left(\omega D_{x}^{\beta} T_{n}^{*}+T_{n}^{*}-b_{1}^{\alpha} T_{n}^{*}\right)\left(x_{1}\right) \\ \vdots & \vdots & \vdots \\ \left(\omega D_{x}^{\beta} T_{0}^{*}+T_{0}^{*}-b_{1}^{\alpha} T_{0}^{*}\right)\left(x_{n}\right) & \cdots & \left(\omega D_{x}^{\beta} T_{n}^{*}+T_{n}^{*}-b_{1}^{\alpha} T_{n}^{*}\right)\left(x_{n}\right)\end{array}\right]$

we can calculate the starting vectors $C_{r}^{0}$, from the initial condition as then

Since $u(0, x)=u^{0}(x)=f(x)$, for all $x \in[0,1]$,

$$
A^{*} C_{r}^{0}=F
$$

\section{Numerical Examples}

Example with known exact solution is considered. The example is chosen such that the behavior of the solution has different characterizations with space and time ranging from polynomial, sinusoidal and exponentially decay. In the calculations, the explicit finite difference methods is used to approximate the fractional time derivative and obtained a fractional boundary value problem along every time level. The solution of the fractional boundary value problem is appro ximated by a truncated shifted Chebyshev polynomials $u^{k}(x)=\sum_{r=0}^{n} C_{r}^{k} T_{r}^{*}(x)$, as a trial solution in the collocation method with the collocation points $x_{j}=\cos \left(\frac{j \pi}{n}\right), j=$ $0,1, \cdots, n$. Also, three graphs have been introduced, in the first $\beta=2$ and different values of $\alpha(0.6,0.7,0.9$ and 1.0$)$, in the second $\beta=1.5$ and different values of $\alpha(0.6,0.7,0.9$ and $1.0)$, and in the third group $\alpha=1$ and different values of $\beta$ ( 1.6, 1.7, 1.9 and 2.0).

Moreover, tables introduced which contain the values of the coefficients of the shifted Chebyshev Polynomial $C_{\alpha, \beta}^{k}$ ( $k$ is the time level $T, \alpha$ is the order of time derivative and $\beta$ is the order of the space derivative). The coefficients are determined along each time step by solving a linear system of equations in the form defined in (22),

Example

Consider the S-TFPDE

$D_{t}^{\alpha} u(x, t)-D_{x}^{\beta} u(x, t)=g(x, t), 0<\alpha \leq 1<\beta \leq 2$; $0 \leq \mathrm{t} \leq 1$ and, $0 \leq \mathrm{x} \leq 1$, where

$g(x, t)=\sin (\pi x) A e^{-t}-e^{-t}\left(\pi^{\alpha} \sin \left(x \pi+\frac{\alpha \pi}{2}\right)\right) ;$

$A=(-1)^{\alpha}$, Subject to the boundary conditions $u(0, t)=u(1, t)=0$, and the initial condition,

$u(x, 0)=\sin (\pi x), 0 \leq \mathrm{x} \leq 1$, whose exact solution is $u(x, t)=e^{-t} \sin (\pi x)$.

Solutions

Consider a trial solution of the form

$$
\begin{gathered}
u_{N}^{k}=\sum_{r=0}^{4} C_{r}^{k} T_{r}^{*}(x)=C_{0}^{k} T_{0}^{*}(x)+C_{1}^{k} T_{1}^{*}(x)+\cdots+ \\
C_{6}^{k} T_{6}^{*}(x)+C_{7}^{k} T_{7}^{*}(x),
\end{gathered}
$$

Now for the first initial solution $u^{0}(x)$ one can calculate it from the initial condition by

$$
u^{k}\left(x_{i}\right)=u^{0}\left(x_{i}\right)=u\left(x_{i}, 0\right)=\sin (\pi x),
$$

$i=0,1,2, \cdots, 7$; where $x_{i}=0.5 * \cos \left(\frac{i \pi}{4}\right)+0.5$, solve $\left[T_{r}^{*}\right]\left[C_{r}\right]^{0}=\left[f\left(x_{i}\right)\right]$, where $r, i=0,1, \cdots, 7$.

The results of the solution of this example, By helping of Matlab are given in the following figures(1-3) and tables (1-3).

\begin{tabular}{|c|c|c|c|c|c|c|c|}
\hline$C_{0.6,2}^{7}$ & $C_{0.7,2}^{7}$ & $C_{0.9,2}^{7}$ & $C_{1,2}^{7}$ & $C_{r}^{0}$ & Col-point & $E_{\beta}^{\alpha}$ & \\
\hline 0.444765568 & 0.43463187 & 0.44526423 & 0.47192077 & 0.472001216 & 1 & $E_{2}^{0.6}$ & 0.075113082 \\
\hline 0.049301693 & 0.02090821 & 0.00373299 & 0.00163802 & 0 & 0.950484434 & $E_{2}^{0.7}$ & 0.062036656 \\
\hline-0.501748138 & -0.46944618 & -0.47150557 & -0.49914043 & -0.49940326 & 0.811744901 & $E_{2}^{0.9}$ & 0.046401034 \\
\hline-0.005010121 & -0.00183968 & $-3.05 \mathrm{E}-04$ & $-1.33 \mathrm{E}-04$ & 0 & 0.611260467 & $E_{2}^{1}$ & 0.009662424 \\
\hline $2.69 \mathrm{E}-02$ & $2.60 \mathrm{E}-02$ & $2.64 \mathrm{E}-02$ & $3 \mathrm{E}-02$ & 0.027992033 & 0.388739533 & & \\
\hline$-3.49 \mathrm{E}-15$ & $-2.08 \mathrm{E}-15$ & $-3.15 \mathrm{E}-16$ & $-3.08 \mathrm{E}-16$ & 0 & 0.188255099 & & \\
\hline$-7.25 \mathrm{E}-04$ & $-6.07 \mathrm{E}-04$ & $-5.66 \mathrm{E}-04$ & $-5.94 \mathrm{E}-04$ & -0.000589991 & 0.049515566 & & \\
\hline
\end{tabular}

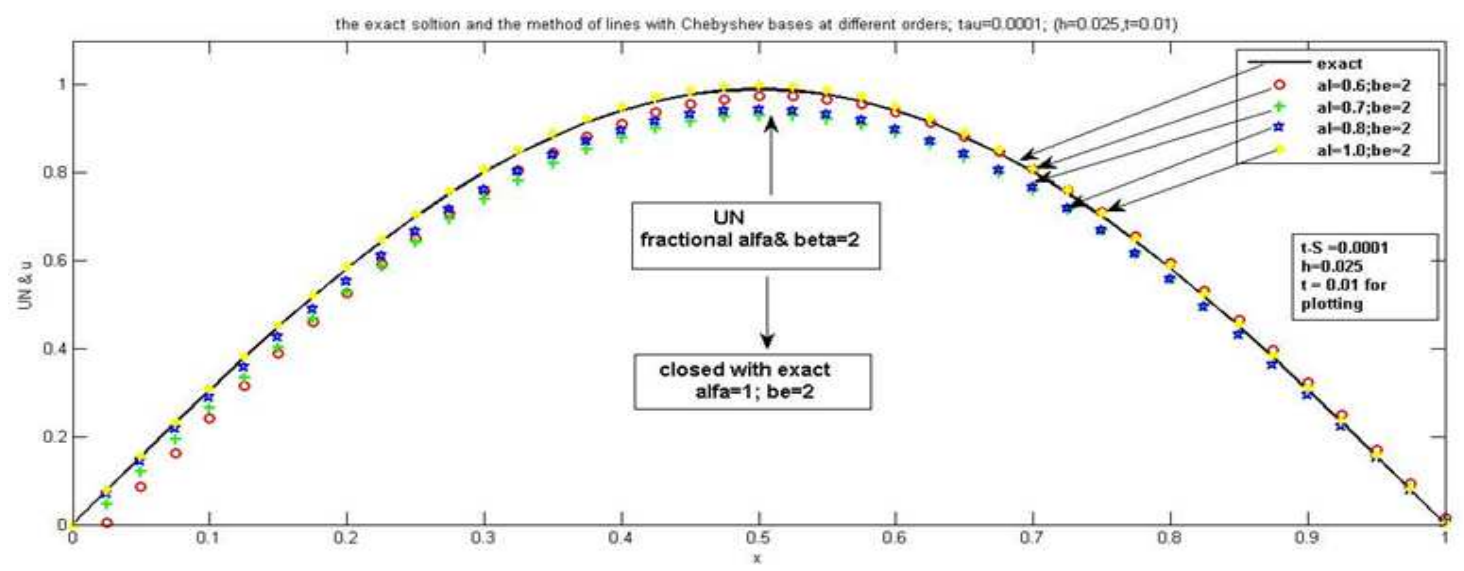

Figure 1. the exact solution and the numerical solution at different orders of $\alpha$ with fixed $\beta=2,(\Delta t=0.0001, n=7), h=0.0125, t=0.01$.

Table 1. $(\Delta t=0.0001, n=7)$. 


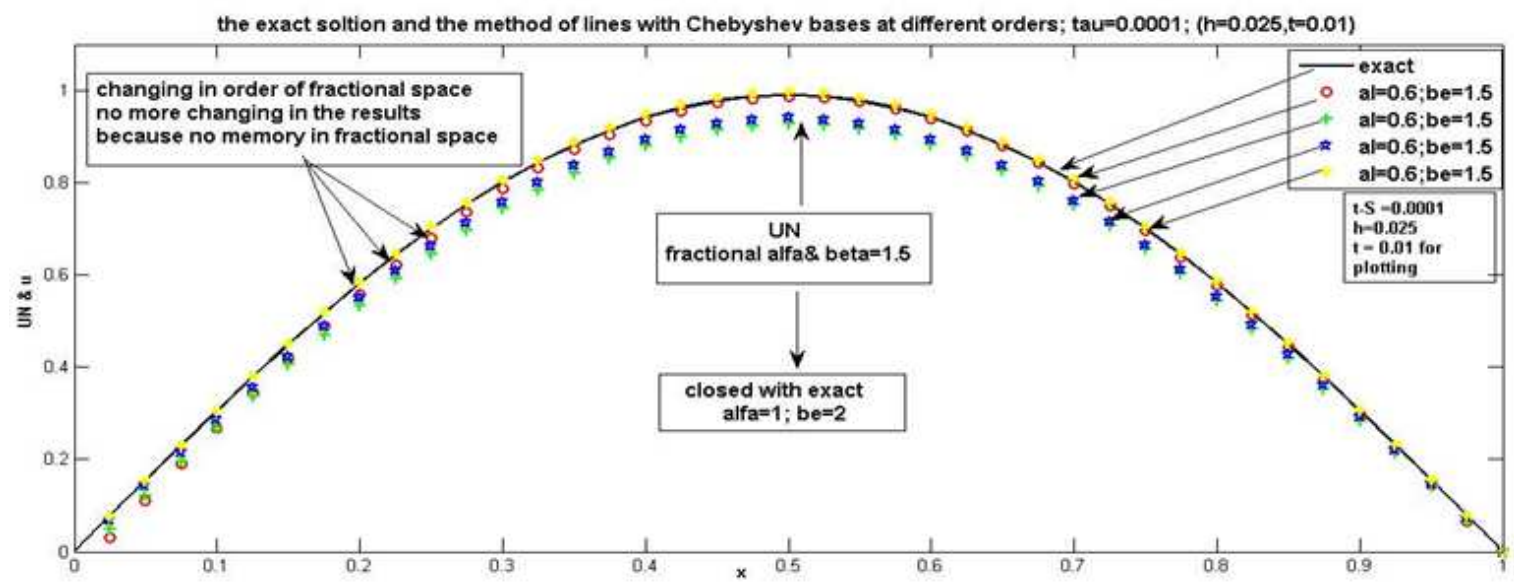

Figure 2. the exact solution and the numerical solution at different orders of $\alpha$ with fixed $\beta=1.5,(\Delta t=0.0001, n=7), h=0.0125, t=0.01$

Table 2. this results at ( $\Delta t=0.0001, n=7)$.

\begin{tabular}{|c|c|c|c|c|c|c|c|}
\hline$\overline{C_{1,1.6}^{7}}$ & $C_{1,1.7}^{7}$ & $C_{1,1.9}^{7}$ & $C_{1,2}^{7}$ & $C_{r}^{0}$ & Col-point & $E_{\beta}^{\alpha}$ & \\
\hline 0.448967636 & 0.42975713 & 0.44202637 & 0.47001453 & 0.472001216 & 1 & $E_{1.6}^{1}$ & 0.007824117 \\
\hline 0.018023305 & 0.01056324 & 0.00272314 & 0.00132116 & 0 & 0.950484434 & $E_{1.7}^{1}$ & 0.008498308 \\
\hline-0.508631601 & -0.47086169 & -0.47122402 & -0.49893334 & -0.49940326 & 0.811744901 & $E_{1.9}^{1}$ & 0.008526541 \\
\hline 7.24E-04 & $3.84 \mathrm{E}-05$ & $-9.21 \mathrm{E}-05$ & $-5.58 \mathrm{E}-05$ & 0 & 0.611260467 & $E_{2}^{1}$ & 0.008457197 \\
\hline 2.83E-02 & 2.64E-02 & 2.64E-02 & $3 \mathrm{E}-02$ & 0.027992033 & 0.388739533 & & \\
\hline$-5.61 \mathrm{E}-04$ & $-3.80 \mathrm{E}-05$ & $-3.80 \mathrm{E}-05$ & $-1.64 \mathrm{E}-05$ & 0 & 0.188255099 & & \\
\hline$-4.60 \mathrm{E}-04$ & $-5.20 \mathrm{E}-04$ & $-5.20 \mathrm{E}-04$ & $-5.73 \mathrm{E}-04$ & -0.000589991 & 0.049515566 & & \\
\hline$-1.65 \mathrm{E}-04$ & $-2.06 \mathrm{E}-05$ & $-2.06 \mathrm{E}-05$ & $-9.31 \mathrm{E}-06$ & 0 & 0 & & \\
\hline
\end{tabular}

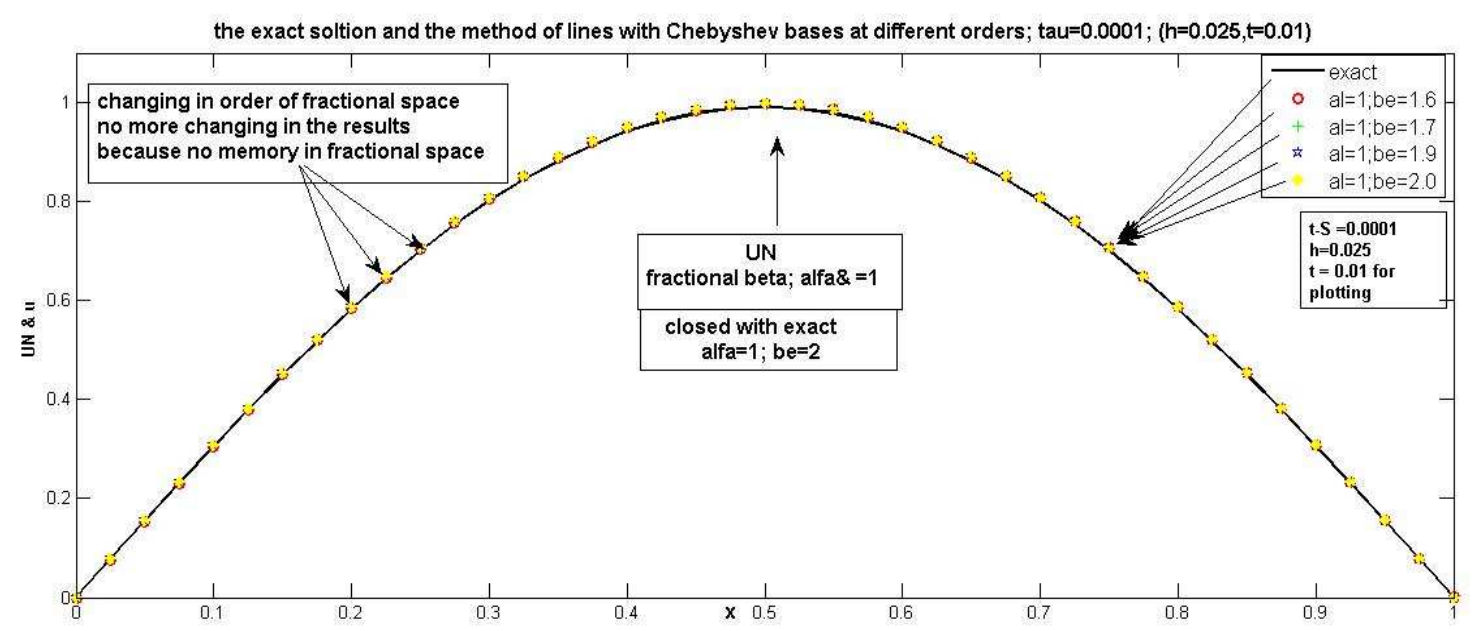

Figure 3. the exact solution and the numerical solution at different orders of $\beta$ with fixed $\alpha=1,(\Delta t=0.0001, n=7), h=0.0125, \Delta t=0.01$

Table 3. $(\Delta t=0.0001, n=7)$.

\begin{tabular}{|c|c|c|c|c|c|c|c|}
\hline$C_{0.6,1.5}^{7}$ & $C_{0.6,1.5}^{7}$ & $C_{0.6,1.5}^{7}$ & $C_{0.6,1.5}^{7}$ & $C_{r}^{0}$ & Col-point & $E_{\beta}^{\alpha}$ & \\
\hline 0.470306567 & 0.47093563 & 0.47094925 & 0.47112135 & 0.472001216 & 1 & $\stackrel{p}{E_{15}^{0.6}}$ & 0.049847529 \\
\hline 0.00147331 & $1.60 \mathrm{E}-04$ & $3.29 \mathrm{E}-0.4$ & $1.48 \mathrm{E}-0.4$ & 0 & 0.950484434 & $E_{15}^{0.6}$ & 0.062972866 \\
\hline-0.498956198 & -0.49907929 & -0.49908717 & -0.49883192 & -0.49940326 & 0.811744901 & $E_{1.5}^{0.6}$ & 0.049849099 \\
\hline$-8.17 \mathrm{E}-05$ & $1.46 \mathrm{E}-0.4$ & $2.09 \mathrm{E}-0.4$ & $-6.75 \mathrm{E}-06$ & 0 & 0.611260467 & $E_{15}^{0.6}$ & 0.007492913 \\
\hline $2.80 \mathrm{E}-02$ & $2.79 \mathrm{E}-0.2$ & $2.78 \mathrm{E}-0.2$ & $3 \mathrm{E}-02$ & 0.027992033 & 0.388739533 & & \\
\hline$-2.93 \mathrm{E}-05$ & $8.94 \mathrm{E}-05$ & $1.81 \mathrm{E}-04$ & $-3.40 \mathrm{E}-08$ & 0 & 0.188255099 & & \\
\hline$-5.60 \mathrm{E}-04$ & $-6.72 \mathrm{E}-0.4$ & $-7.66 \mathrm{E}-04$ & $-5.90 \mathrm{E}-04$ & -0.000589991 & 0.049515566 & & \\
\hline$-1.56 \mathrm{E}-05$ & $4.00 \mathrm{E}-0.5$ & $8.75 \mathrm{E}-05$ & $-1.49 \mathrm{E}-07$ & 0 & 0 & & \\
\hline
\end{tabular}

\section{Discussion and Conclusions}

It is known that many differential equations can be solved by using the WRM. The collocation method is the simplest method among the WRM. The efficiency of using this method depends on the selection of the collocation points. The treatment depends on transforming the time dependent parabolic FPDE into a family of fractional boundary value 
problems along time levels with the help of the finite difference method. the shifted Chebyshev polynomials of the first kind is used as the bases function of the trial solution, and the Gauss Lobatto points are used as the collocation points., thus the problem is transformed to one of solving an algebraic linear systems in the coefficients of the bases functions included in the trial solution. Thus an explicit approximation is obtained for the solution along each time level which enables to approximate the solution at any point along this time level not only at some grid points as in the finite difference. Although the algebraic system is dense in this treatment in comparison with those obtained with the finite difference method the number of equations is relatively very small. From the graphs one can see that this treatment performs more efficiently in the integer case (as the fractional orders of the derivatives approaches the classical integer case as the approximate solution approach the exact solution).

\section{References}

[1] G. Caporale, and M. M. Cerrato, Using Chebyshev Polynomials to Approximate Partial Differential Equations, Comput Econ , 2010.

[2] B. Costa, Spectral Methods for Partial Differential Equations, A Mathem-Journ, Vol. 6, December, 2004.
[3] Z. C. Kuruo $\breve{g l u, ~ W e i g h t e d-r e s i d u a l ~ m e t h o d s ~ f o r ~ t h e ~ s o l u t i o n ~ o f ~}$ two-particle Lippmann-Schwinger equation without partial-wave decomposition, rXiv, physics.comp-ph, 17 Jul, 2013.

[4] N. Nie, J. Huang, W. Wang, and Y. Tang, Solving spatial-fractional partial differential diffusion equations by spectral method, Journal of Statistical Computation and Simulation, 2013

[5] S. A. Odejide, and Y. A. S. Aregbesola, applications of method of weighted residuals to problems with semi-infinite domain, Rom. Journ. Phys., Vol. 56, Nos. 1-2, P. 14-24, Bucharest, 2009.

[6] R. K. Rektorys, "The method of discretization in time and partial differential equations", Springer Netherlands, Dec 31, 1982.

[7] Li. Xianjuan, and Xu. Chuanju, A Space-Time Spectral Method for the Time Fractional Diffusion Equation, This work supported by National NSF of China , High Performance Scientific-Computation Research Program CB321703, 2005.

[8] J. P. Boyd, Chebyshev and Fourier Spectral Methods, Second Edition, University of Michigan, Ann Arbor, Michigan 48109-2143, 2000.

[9] M. Dalir, and M. Bashour, Applications of Fractional Calculus, App- Math- Scie- Vol. 4, 1021 - 1032 , 2010. 